\title{
Family Skills Training Intervention for ADHD: Putting the Child Back into Child Therapy
}

David F Curtis ${ }^{1 *}$, Corey L Heath ${ }^{1,2}$, Stephanie G Chapman ${ }^{1}$ and Sara R Elkins ${ }^{1}$

${ }^{1}$ Department of Pediatrics, Psychology Section, Baylor College of Medicine and Texas Children's Hospital, Houston, Texas, USA ${ }^{2}$ Department of Educational Psychology, University of Houston, Houston, Texas, USA

\begin{abstract}
Attention-Deficit/Hyperactivity Disorder (ADHD) affects over $7 \%$ of school-aged children in the US, and it is the most common reason pediatricians refer children for psychosocial treatment. However, the range of effective treatments for ADHD is limited to four interventions (i.e., medication, Behavioral Parent Training (BPT), behavioral classroom modifications, intensive summer programs). Notably, current behavioral interventions indirectly treat disruptive behaviors associated with ADHD through parental participation and often don't reduce core ADHD symptoms of inattention and hyperactivity/impulsivity. While multiple dimensions of attention problems are associated with ADHD, our understanding of these functional impairments has yet to guide new approaches to intervention. The purpose of this paper is to introduce a model for innovative, child-focused approaches to ADHD intervention. First, we discuss the multidimensional impairments of attention that are associated with ADHD (e.g., behavioral activation, inhibition, and adjustment problems) in order to guide the development of specific operational targets and intervention strategies for child-focused behavioral intervention. Next, we exemplify these novel methods within complementary child-focused therapy that can be delivered in conjunction with BPT; thereby, increasing the magnitude and timeliness of its effects. Finally, we illustrate how development of a child-focused intervention for ADHD promotes opportunities to reach more children in need across a variety of settings. Implications for expanding clinic-based standards of care are discussed as well as innovative practices for addressing ADHD-related problems in school and primary care settings.
\end{abstract}

Keywords: ADHD; Child-focused intervention; Behavioral parent training; Family skills training

\section{Introduction}

Attention-Deficit/Hyperactivity Disorder (ADHD) is one of the most common behavioral health concerns in children and adolescents, with a prevalence rate of $2 \%$ to $9 \%$ worldwide [1]. Children with ADHD present with executive functioning impairments and behavioral challenges that negatively affect their daily functioning across everyday settings [2]. These difficulties place them at greater risk for suffering adverse outcomes throughout the life span, including disruptions in relationships (with parents, peers, and teachers), academic problems, chronic behavioral difficulties, and delinquency and substance abuse in adolescence $[3,4]$.

At this time there are only four empirically-based treatments considered to be best practices for treating ADHD: medication, Behavioral Classroom Management (BCM), Behavioral Parent Training (BPT), and behavioral peer interventions in recreational settings [5-7]. While each of these may be incorporated within a more comprehensive treatment plan, a major limitation of psychosocial interventions is that they predominantly work with adult caregivers without fully incorporating the participation of the child with ADHD. Thus, treatments tend to focus on the child as the beneficiary of behavioral strategies, but fail to directly engage the actual patient to promote better self-management skills or coping strategies. The most common psychosocial interventions - Behavioral Classroom Management (BCM) and Behavioral Parent Training (BPT) - are useful in minimizing behavioral disruptions related to ADHD, but have been criticized for failing to produce reliable behavioral change in core ADHD symptoms that generalizes across time and settings $[7,8]$. Finally, summer treatment programs provide exemplary models of integrated services for parent training, child skills training, and medication management; however, these interventions may be hard to access due to geographic limitations in availability and the seasonal nature of the programming [9].

ADHD is one of the few behavioral health conditions for which direct, child-focused therapies have not been successful [6]. To understand why child-focused treatments have not been effective, a key question to consider is what makes ADHD so hard to treat? It is likely that the impaired attention systems in ADHD that negatively affect self-regulation also interfere with typical experiences within insightoriented individual therapies. Cognitive-Behavioral Therapy (CBT) is arguably the psychotherapy approach that has demonstrated the greatest effectiveness for a large number of childhood conditions [10]. However, inconsistent performance in planning, self-monitoring, and meta-cognition (considering consequences of actions ahead of time) is an inherent deficit to ADHD [11,12]. These areas of functioning are necessary to benefit from CBT, which typically aims to promote better behavioral inhibition (e.g. self-monitoring, suppressing responses to negative thoughts) and targets distorted thinking [10]. Problems associated with ADHD involve regulation of thought processes (e.g. planning and sequencing information) rather than distortions of thought content [13]. Thus, ADHD is most notably (1) a disorder of performance, not ability, and (2) a cluster of problems related to thought processing, not thought content $[4,14]$. Consequently, the efficacy of individual CBT approaches for treating child ADHD symptoms has not been supported by research findings $[6,7,15]$.

Recent research advances have transformed our understanding about the relations between behavioral, neural, and cognitive impairments observed in children with ADHD [14,16]. Behavioral inhibition and activation systems have long been implicated with regard

*Corresponding author: David F Curtis, Department of Pediatrics, Psychology Section, Baylor College of Medicine and Texas, Children's Hospital, Houston, Texas, USA, Tel: +1 832-822-3700; E-mail: dfcurtis@texaschildrens.org

Received July 23, 2013; Accepted December 04, 2013; Published December 06, 2013

Citation: Curtis DF, Heath CL, Chapman SG, Elkins SR (2013) Family Skills Training Intervention for ADHD: Putting the Child Back into Child Therapy. J Psychol Abnorm Child 2: 107. doi:10.4172/2329-9525.1000107

Copyright: $\odot 2013$ Curtis DF, et al. This is an open-access article distributed under the terms of the Creative Commons Attribution License, which permits unrestricted use, distribution, and reproduction in any medium, provided the original author and source are credited. 
to their functional roles in behavioral self-regulation, a fundamental impairment for children with ADHD [17]. Proximal neural mechanisms within the prefrontal cortex and striatum have also been associated with ADHD impairments based upon numerous studies using fMRI procedures [18-20]. Further, a recent study by Dennis and colleagues has even discriminated ADHD impairments from several other neurobehavioral conditions by establishing a clear cognitive phenotype for the disorder [11]. Combined, these studies of behavioral self-regulation, neural mechanisms, and cognitive phenotypes clearly illustrates that attention problems are multidimensional in nature. While often characterized as a disorder of response control, ADHD-related problems with attention extend beyond impaired behavioral inhibition to include difficulties with cognitive activation (e.g. divided attention, sustained attention) and cognitive adjustment (self-monitoring/ appraisal of performance, restraint from attending to distractions) [11]. Thus, ADHD-related impairments go far beyond whether or not a child can simply pay attention.

Considering this broader conceptualization of attention problems, new behavioral methods are needed to operationalize multiple elements of attention and relate these processes to behavioral functioning. Psychosocial approaches to treating ADHD have typically applied CBT methodology known to be effective for other childhood conditions, but in ways that are insight-oriented and focusing on improving inhibitory control rather than considering other core aspects of attention such as activation of behaviors and cognitive adjustment to behavioral demands. In considering the function of these ADHD neurocognitive impairments, the techniques used in child-focused CBT may certainly be helpful if applied in different ways to meet the specific needs of this clinical population. Accordingly, the content of ADHD child therapy may be of less importance than the process imposed. Consequently, "what" we do in child therapy for ADHD may be less important than, "how" we do it [21].

The primary purpose of this paper is to discuss processes and modalities for, "putting the child back into child therapy" for ADHD. In doing so we aim to illustrate how complementary child-focused intervention methods can be used to treat ADHD, based upon a multidimensional view of attention-related impairments known to be associated with the disorder. We offer specific types of child-focused behavioral interventions for ADHD that are organized to address these core, underlying features of attention problems as outlined by Dennis and colleagues' taxonomy of attention systems (see Table 1 in Appendix A). However, the goal of this paper is not provide a direct translation of neurocognitive mechanisms to intervention, but rather to illustrate how this research may inform our clinical practice by allowing us to develop better ways to engage children directly in their own care for ADHD-related problems.

\section{Advantages of Child-focused Intervention for ADHD}

Since studies of behavioral parent training and classroom behavioral modifications have demonstrated moderately large effect sizes $[6,7]$, why should we even consider developing child-focused approaches for ADHD? There are several potential child and parent benefits for complementing traditional care for ADHD with a child-focused intervention. Child participation in therapy is arguably important to parents for several reasons. Parents seeking intervention for their child's ADHD often preconceive behavior therapy to be a childfocused experience. It is often difficult for parents to understand why their participation in therapy is highlighted with less attention given directly to their child diagnosed with ADHD. Parents may also present with a less sophisticated understanding of their child's diagnosis, the functional impairments and chronicity of these problems implicated, and how ADHD symptoms may limit their child's independent use of skills presented in therapy. Parents are also likely to be unaware of best practices established for ADHD and the proven effectiveness of parent-focused behavioral intervention [6,7]. As a result, they may feel a greater need for the clinician to spend more direct time with their child in order to better understand his/her individual symptoms and needs. Doing so within a child-focused milieu would attend to these needs, while also allowing parents to "warm up" to the necessity of their role in the child's therapy without implicitly feeling blame for the child's presenting problems.

There are also many advantages from the child's perspective for providing child-focused objectives for ADHD intervention. First, including child participation in behavior therapy explicitly conveys (to parents and children) the notion that the child is capable of making a meaningful contribution to the process. In contrast, not including child-focused objectives in therapy implicitly conveys a diminished appreciation for the child's role. A second child benefit is the opportunity for the therapist to better assess and understand the child's strengths and interests in order to harness his/her motivation to participate in clinical, home, and school-based treatment activities. Children with ADHD often present with diminished self-esteem and emerging or comorbid symptoms of oppositionality or conduct related problems due to their frequent experience of redirection, correction, and punitive measures [22]. Increasing their motivation within a child-focused milieu offers the advantage of establishing an effective - "participation principle" for implementing new behavioral strategies [23]. Third, child-focused objectives promote opportunities for increasing child competencies with therapist also being able to clearly observe and relate specific feedback back to the child and his/her parents to provide evidence of target behaviors performed. This behavioral rehearsal and competency development achieves two goals. It promotes greater child confidence and self-efficacy for performing these tasks in front of parents and others. It also allows the therapist to convey these observations to parents, thereby promoting more positive self-esteem for the child within the family context and modeling (for parents) the delivery of specific labeled praise. Finally, child-focused intervention allows the clinician to prime the child to participate more effectively in family session rehearsals and home implementation of new strategies. This priming allows the clinician to more effectively intervene at a family systems level, providing the child with clear expectations for new parenting behaviors (and appropriate child responses) and pre-emptive problem-solving opportunities to work through embedded parentchild interaction patterns within the context of the family session. In considering child-focused therapy for ADHD, these treatment objectives can be implemented across a variety of treatment modalities. In the sections to follow, we illustrate how child-focused therapy can be implemented alongside behavioral parent training to complement usual care.

\section{Complementary Child-focused Therapy}

For all the reasons outlined above, we believe that adding a childfocused behavioral therapy as a complement to behavioral parent training presents great utility for increasing and sustaining the behavioral treatment gains for children with ADHD. The literature suggests that behavioral parent training is conducted either exclusively with parents or within a family therapy milieu, but with therapy objectives directed predominantly toward parents [5]. Similar to behavioral parent training, our proposal for a move in the field towards Family Skills Training recommends an intervention that provides parent-focused behavior management skills, while also incorporating 
child-focused intervention strategies and integrating child and parent focused strategies within a family milieu [24]. Family Skills Training (FST) can carry out these treatment objectives in a number of ways. The general treatment objective for FST is to improve parent and child self-efficacy for managing ADHD-related disruptive behaviors across home, school, and social settings. Although intended to be discussed only theoretically in this paper, it is useful to note that the model of Family Skills Training has been employed within the Family STARS (Family Skills Training for ADHD-Related Symptoms) program, a 10-session, manualized approach that we will discuss briefly in order to illustrate the goals of FST $[24,25]$.

The parent training component of Family STARS gives special attention to the role of ADHD in managing child disruptive behaviors. In doing so, Family STARS integrates many of the common components of well-established and widely available parent training programs, including those developed by Barkley, Cunningham, McMahon and Forehand, and Sanders [26-30]. Session content is closely coordinated with the materials planned for complementary child-focused sessions, which utilize a strengths and mastery-based approach. In terms of specific session content, FST is structured to first provide psychoeducation about ADHD followed by strategies to improve structure and routines at home, parent facilitation of childdirected interaction, implementation of a positive reinforcement system, positive methods for correction and redirection, and finishing with generalization and behavioral relapse prevention methods [24].

The child therapy component of Family STARS is designed to complement the work simultaneously conducted within the behavioral parent training session. In contrast to traditional CBT methods for child intervention, little background rationale is provided to child participants and the focus of sessions is not to build child insight. Instead, child sessions are structured and implemented with great heed given to multiple dimensions of attention problems and the high frequency demands often prompting difficulties for children with ADHD. Key components of the child therapy include the use of (1) consistent, stable in-session routines, (2) experiential, rather than didactic learning opportunities, (3) benchmarking strategies to improve child motivation to engage in targeted behavioral rehearsal, and (4) over-learning principles to promote more frequent child practice and greater mastery of skills for key target behaviors. These key therapy components can be observed across the structure, process, and content of the Family STARS child therapy.

Given the tendency of children with ADHD to perform best in environments that are highly structured, child routines are consistently applied across sessions. Each child session routine generally involves the introduction and frequent rehearsal of a target behavior, review of the weekly home implementation of therapy assignments and behavioral strategies learned to date, and introduction of new behavioral strategies (e.g. organizational strategies for academic materials, behavioral strategies for coping with everyday stressors, model-driven scripts for accepting consequences, etc.) that will be intensively reinforced by parents throughout the upcoming week. Child session content includes priming for home implementation of parent-led strategies (such as daily goals, the token economy and time-out) that are being introduced concurrently within the parent session. Finally, child sessions culminate with a transition to the parent session for integrated family rehearsal of new behavioral methods introduced.

Process elements of the child therapy include activities that intensively promote over-learning of desired behaviors commonly associated with high frequency demands (e.g. verbal and nonverbal attending skills). Child sessions emphasize experiential learning that includes verbal description, social modeling of target behaviors, intensive child rehearsal of target behaviors, and clinician feedback and reinforcement for behaviors performed. Through the use of over-learning techniques, children engage in repeated rehearsal of target behavioral responses with the purpose of priming automatic adaptive responses to common, everyday situations. Therapists utilize benchmarking strategies to provide in vivo reinforcement and increase child motivation for practicing target behaviors. Benchmarks are child determined and personalized based upon the individual's performance within the previous session. Once a target behavior is established in session, the therapist monitors the frequency of child performance using a tally counter. The therapist displays demonstrative clicks of the tally counter and delivers specific verbal praise when target behaviors are observed. This not only marks the point of performance for the behavior, but also provides ongoing feedback to the child for adjusting his/her behaviors to maximize potential rewards. Emphasis is placed on verbal praise in these instances and discussion of the actual frequency count is deferred until the end of the session. This minimizes distraction by preventing the child from focusing on the number of clicks instead of the behavior being reinforced.

The content of child-focused sessions maps closely to each of the parent sessions, and is designed to prepare children for home and school implementation to be implemented by parents. Child sessions introduce the same behavioral strategies given to parents (e.g. child directed interaction, token economy, time-out, etc.), but are framed in a manner that is developmentally accessible for the child and in a way that actively promotes the child's role in collaborating with parent-led methods. Core objectives for Family Skills Training (FST) child sessions are outlined in Table 2 (Appendix B).

\section{Family Skills Training Modalities}

One of the biggest advantages of having a manualized protocol for Family Skills Training (FST) is that it can be delivered effectively across a variety of treatment modalities with different clinician configurations. The Single-Family Intervention delivers the intervention to a single family with 2 clinicians dividing roles across parent and child sessions. A Modified Single-Family Intervention can also be used with a single clinician, but with the session divided into child-focused and parentfocused modalities. The Multi-Family Intervention allows for a group of parents to meet within a workshop/support group environment, while children are paired and work in dyads in child-focused therapy. Decisions regarding which treatment modality to choose can be made after carefully considering factors such as clinical space, appointment timeslots for peak therapy times requested (e.g. after school, evenings, weekends) therapist availability, third-party payer authorization, and patient preferences.

\section{Single-family Intervention}

This format engages a single family in behavior therapy, working with two therapists, one implementing the child-focused intervention and the other providing behavioral parent training. Within this format, the parents and their child will attend separate, but adjacent therapy sessions for the first 35-45 minutes of the session. When all of the child-focused therapy objectives have been achieved, the child therapist transitions with the child to join his/her parents for an integrated family consultation. While the child is debriefing his/her parents about the activities of the child session, this transition provides a natural break in the session that enables the two treating clinicians to excuse themselves and briefly consult one another regarding key needs 
for in-session behavioral rehearsal. In clinics equipped with one-way mirrors, a reflecting team approach may also be incorporated to add other team members' observations and feedback for integrating the two concurrent sessions. The family session will then culminate by utilizing the remaining 10-15 minutes for therapist modeling, whole family behavioral rehearsal, troubleshooting, and administering of token rewards. While utilizing more therapy personnel than other modalities, this FST format provides the benefit of an intensive, integrated approach, potentially enabling the family to achieve treatment goals within a shorter time-frame.

\section{Modified Single-family Intervention}

In most cases, only one therapist may be available to provide Family Skills Training. In these circumstances, a modified version of the SingleFamily Intervention can be utilized. Within this structure, the treating clinician conducts a brief child-focused session at the beginning of the family visit, establishing and reinforcing child-focused treatment objectives and target behaviors to perform within the family session. In the latter part of the session, the clinician prompts the child to convey his/her current target behaviors. This is followed by therapist modeling the delivery of positive reinforcement with specific, labeled praise and clicks of the tally counter for each display of the target behaviors. The session then focuses upon more typical behavior management strategies for parents, but proceeds in a more integrated fashion with parents working actively with their child to discuss, observe therapist modeling, and rehearse new strategies for home implementation. The clinician also continues to apply benchmarking to monitor and reinforce the child's target behaviors displayed in-session. This allows the child to develop more adaptive behavioral responses and confidence for everyday demands, while modeling effective reinforcement of target behaviors for parents to emulate within the home setting. An obvious benefit of the Modified Single-Family Intervention is the limited demand placed upon personnel resources, as only one therapist is engaged in service provision with the family. Additionally, this modality allows for more frequent modeling and facilitation of family rehearsal for positive communication and interaction strategies, with the support and guidance of the therapist.

\section{Multi-family Intervention}

The Multi-Family Intervention is a group-based treatment approach provides parents and children with separate, complementary therapy delivery. Multi-Family Intervention delivers behavioral parent training within a group parenting workshop format, while at the same time providing child-focused therapy in dyads with a child therapist. The multifamily format includes all the previously outlined therapy procedures and content, but facilitates opportunities for social learning of behavioral strategies and interpersonal support for coping with ADHD-related challenges. As illustrated in Cunningham's group parenting intervention for ADHD [27], parents within the MultiFamily Intervention group gain validation and normalization of parenting challenges related to having a child with ADHD as well as an understanding that their child is not the only one experiencing such problems.

Child participants also gain the opportunity to work with a partner (also struggling with ADHD) for the duration of treatment, allowing for increased social support, opportunities to normalize the ADHD diagnosis, and a milieu for practicing socially relevant target behaviors within each session (such as interrupting appropriately and respecting personal space). Contrary to possible iatrogenic effects observed in larger group therapies for children with disruptive behavior problems [31], this structured, dyadic, behavior therapy provides social facilitation of therapy goals within a tightly controlled milieu. The dyadic milieu provides unique opportunities to correct problematic child behaviors as they occur in-session. Noncompliance with the target behavior is addressed by using methods such as differential attention or cue-controlled redirection. Using differential attention, the clinician removes all attention from the child who is off-task and enthusiastically directs his/her attention to the child's partner who is complying with the target behavior. In doing so, the clinician delivers specific, labeled praise to both reinforce the on-task child and to prompt - "self-redirection" for the child who is off-task by clearly modeling how to gain positive attention. Conversely, the clinician may use cuecontrolled responding to redirect problem behaviors (e.g., prompting children to stop what they are doing by saying 'Freeze!' and return to their seats by saying (Melt!'). Another way this can be done is by using a key word to elicit a very specific child verbal response, which prompts them to return to their seats.

While children are given an opportunity to learn through social observation of their peers being successful, they also benefit from the opportunities to work collaboratively with another child who also experiences ADHD-related challenges. In the dyad milieu children are required to work together, rather than against one another, when attempting to exceed their behavioral benchmark from the previous week. This social facilitation promotes collaborative work between the children and allows for differences in ability and/or motivation each session to be compensated for by cumulative ratings. Children are also provided with behavioral goals (such as "encouraging others" or "cooperating" designed to promote social collaboration and development of appropriate social skills. In this way, children are provided with clear expectations for high-frequency target behaviors and are given numerous opportunities to practice these skills and observe a socially valid peer model over multiple sessions.

\section{Implications of Child-focused Objectives for the Treatment of ADHD}

There are a number of important factors that affect the adoption and sustainability of child treatments for ADHD across healthcare settings. In evaluating the treatment effectiveness of child-focused interventions for ADHD, it is important to consider whether these treatments may be efficiently and appropriately implemented by providers, effective and acceptable to children and families, and supported by third-party payers [32]. Chorpita outlined three essential elements that are needed for an intervention to be deemed effective; these include an intervention's feasibility, generalizability, and its expected benefits [33].

\section{Feasibility}

The feasibility of an intervention refers to its capability of being implemented within a true practice setting. Treatment feasibility encompasses its acceptability (for clinicians and their patients), it's potential to achieve implementation fidelity, and the possible trainability of its methods [33]. Child-focused approaches to ADHD intervention are not only implemented easily within most mental health settings, they also offer versatility to expand the modalities of usual care models of treatment [34].

The acceptability of child-focused interventions to parents, children, and providers is critical to its broad implementation [24,32]. Parents' ratings of acceptability and perceived effectiveness for interventions that include a child component are generally higher than those with only a parent focus [35]. Parents may also hold misperceptions about the structure of treatment for ADHD, since behavioral parent training 
is not the primary evidence-based treatment for many other childhood psychological conditions. A family's acceptability of a treatment is often most clearly seen via treatment drop-out rates. In this regard, Family Skills Training may facilitate greater treatment engagement of families, as inclusion of a child-focused component of therapy would be expected to increase treatment acceptability for families.

It is also likely that new child-focused objectives for treatment would be well accepted by therapists. Community clinicians continue to provide individual therapies to children with ADHD even though these treatment approaches have been proven to be ineffective for ADHD intervention [36]. Rather than extending existing models of individual psychotherapy (e.g. cognitive-behavioral therapy), known to be ineffective for ADHD, additional clinician training would be required to implement the proposed child-focused behavioral approaches to intervention. Training difficulties have been reported as major barriers to clinician adoption of novel clinical procedures [32,33]. Utilizing a well-defined and structured treatment protocol for these child approaches allows these methods to be "packaged" to maximize accessibility for clinician training. Thus, the novel childfocused interventions proposed here have the potential to effectively be used in individual treatment modalities as the evidence-base for these interventions grows.

\section{Generalizability}

The potential effectiveness of child-focused intervention approaches for ADHD is also dependent upon their generalizability. This considers not just whether an intervention can be feasibly implemented, but how well it works outside the control of a clinical laboratory setting [33]. Though yet to be tested outside the clinical research setting, child-focused behavioral approaches will likely be more generalizable than current standards of care. Behavioral Parent Training (BPT) and classroom behavioral modification require the participation of parents and teachers who may face time constraints or logistical barriers that prevent them from regularly attending therapy or behavioral planning meetings. However, child-focused intervention approaches present ways to circumvent these potential barriers by their transportability by extending service delivery within alternative treatment settings such as schools.

Another way that child-focused behavioral intervention for ADHD can promote greater generalizability is by making treatment more accessible to consumers. For instance, the multifamily format of Family Skills Training enables several families to be treated at the same time. After school and after work clinic times are often limited, creating longer waitlists for families seeking intervention within a restricted range of times. Multifamily intervention not only allows many more patients to be seen during these primetime hours, but also provides an intensive intervention model that incorporates social learning and interpersonal support.

\section{Expected Benefits}

Since ADHD-related problems are the most commonly referred needs for psychological intervention [4], the cost-effectiveness of ADHD intervention cannot be overstated. In fact, Pelham, Foster and Robb estimate that (on average) each child with ADHD incurs between $\$ 12,500-\$ 17,458$ in incremental costs each year related to health and mental health needs, educational supports, crime and delinquency, and family costs such as work loss [37]. Although there have been no formal cost-effectiveness analyses conducted on the proposed child interventions for ADHD, the framework for such interventions yields promising benefits. For example, outcome studies of the Family Skills
Training protocol indicated a larger effect size (Cohen's $d=1.00$ ) [24] than currently published effects for traditional behavioral parent management interventions ( $d=0.70$ for comparable pre-post studies) [6]. Though this Family Skills Training study used two therapists for each family, treatments were implemented without any participant drop-outs and with similar or shorter dose response (10 sessions) [24] than those currently published (e.g. range $=8-11$ sessions, [6]; 1222 sessions, [38]). Thus, the mean cost of treatment for these families was likely significantly lower than families participating in longer-term treatments for ADHD.

The use of child-focused treatment approaches may also enable behavioral services to be delivered in a manner that is cost-effective to clinicians and their systems of care. Calling again upon the example of a multifamily intervention that employs BPT with complimentary child therapy, parent training can be delivered to a cohort of parents by a single therapist while other child clinicians carry out child-focused, pair therapies. Based upon this algorithm, six families would require only four therapists, eight families-five therapists, and so on. Finally, child-focused protocols could also be implemented in school settings without the need for direct parent participation, thereby reducing societal costs due to parent work loss, health care usage, etc. [37].

\section{Potential Barriers and Limitations for Child-focused Therapies for ADHD}

Although the implications of utilizing child-focused interventions for ADHD are evident, several limitations should be considered in determining the effectiveness of such interventions. First, personnel requirements for implementation of child-focused treatment strategies complementary to BPT may stress the capacity of a clinic by depending upon the use of many therapists at the same time. For example, to provide single family BPT+child behavioral activation therapy would require two clinicians per family. In addition to personnel costs, inconsistencies in third party authorization and reimbursements for care may impact the likelihood that these forms of treatment can be provided in many settings. At present, multifamily therapy is often not reimbursed by third-party payers. Similarly, because complementary child-focused intervention within a BPT training modality is currently unprecedented, use of Current Procedural Terminology (CPT) codes require some degree of interpretation (e.g. should intervention be billed via a family or an individual psychotherapy code?).

\section{Future Directions}

Despite encouraging evidence for the effectiveness of interventions for ADHD that target the individual child, there are many unanswered questions important for the development and refinement of these interventions. Further research is needed to enhance our understanding of the effectiveness of child-focused treatments for ADHD.

Research on child-focused treatments that address core symptoms of ADHD has the potential to offer a great impact on the field of ADHD treatment. Existing psychosocial treatments for ADHD involve parent and teacher-focused strategies, which promote child behavior change indirectly via environmental engineering. Notably, these approaches tend to promote greater reduction in associated disruptive behaviors (e.g., compliance, aggression, and emotion-regulation) than core symptoms of ADHD [39]. Thus, future research examining approaches that directly target child behaviors to reduce core ADHD symptoms is needed to gain more definitive evidence for the effectiveness of childfocused treatments. In this regard, it is clear that implementation of child-focused strategies for ADHD will require more rigorous study to examine potential mediators of the relationship between social leaning 
based intervention and neurocognitive processes for children with ADHD.

Similarly, future work should aim to identify moderators of the relationship between child focused intervention and outcome to determine for whom treatment is most effective. This work should examine whether outcomes are improved for younger versus older children, single-parent versus dual-parent households, and those with less or more severe symptoms or comorbidities. At present we know that combined medication and behavioral treatments for ADHD work for a little more than two-thirds of children (68\%), leaving the remaining one-third with significant attention and behavior problems [40]. It may be that implementing child focused interventions as an adjunct to behavioral parenting training can enhance treatment effects for children who have previously been unresponsive or minimally responsive to traditional behavioral parent training and/or medication treatment approaches. These kinds of examinations may also shed light on protective factors that could prevent or ameliorate commonly cooccurring problems such as social difficulties or mood disturbance that often result from children frequently encountering negative environmental feedback related to their ADHD symptoms (e.g., being identified as problem-child, excluded from social activities by others, frequent teacher corrections, etc.).

In closing, we believe that children with ADHD can make positive and meaningful contributions to their own treatment. However, with the exception of intensive summer treatment programs, effective utilization of child participation in their own ADHD treatments represents a significant absence within the empirical literature. Childfocused treatment approaches that are guided by our knowledge of neurocognitive impairments for children with ADHD may help children develop better strategies for addressing common everyday demands. Involving children in their own treatment for ADHD creates opportunities to extend treatment gains beyond a time-limited therapy. Further, implementation of a child-focused treatment approach may improve the accessibility and transportability of behavioral treatments, making them more widely available to families who may be otherwise limited by parents' inability to participate. Distinct benefits may exist for both parents and children when participating in a treatment approach that includes direct child participation. While such programs will benefit from continued investigation to better understand their relative advantages, we believe that inclusion of children in their own treatment represents emerging innovation in behavioral treatments for ADHD.

\section{References}

1. Froehlich TE, Lanphear BP, Epstein JN, Barbaresi WJ, Katusic SK, et al. (2007) Prevalence, recognition, and treatment of attention-deficit/hyperactivity disorder in a national sample of US children. Arch Pediatr Adolesc Med 161: 857-864.

2. National Institute of Mental Health [NIMH] (2008) Attention deficit hyperactivity disorder (ADHD) U.S. Department of Health and Human Services, National Institutes of Health (NIH Publication No. 08-3572) Author: Washington, DC, USA.

3. Barbaresi WJ, Colligan RC, Weaver AL, Voigt RG, Killian JM, et al. (2013) Mortality, ADHD, and psychosocial adversity in adults with childhood ADHD: a prospective study. Pediatrics 131: 637-644.

4. Barkley R.A. (2006) Attention deficit hyperactivity disorder: A handbook for diagnosis and treatment. Guilford: New York, USA.

5. Diamond G, Josephson A (2005) Family-based treatment research: a 10-year update. J Am Acad Child Adolesc Psychiatry 44: 872-887.

6. Fabiano GA, Pelham WE Jr, Coles EK, Gnagy EM, Chronis-Tuscano A, et al. (2009) A meta-analysis of behavioral treatments for attention-deficit/ hyperactivity disorder. Clin Psychol Rev 29: 129-140.

7. Pelham WE Jr, Fabiano GA (2008) Evidence-based psychosocial treatments for attention-deficit/hyperactivity disorder. J Clin Child Adolesc Psychol 37: 184214.

8. Valdez C, Carlson C, Zanger D (2005) Evidence-based parent training and family interventions for school behavior change. School Psychology Quarterly 20: $403-433$.

9. Coles EK, Pelham WE, Gnagy EM, Burrows-MacLean L, Fabiano GA, et al (2005) A controlled evaluation of behavioral treatment with children with ADHD attending a summer treatment program. Journal of Emotional and Behaviora Disorders 13: 99-112.

10. Kendall P (2011) Child and adolescent therapy: Cognitive-behavioral procedures. (4thnedn), Guilford: New York, USA

11. Dennis M, Sinopoli KJ, Fletcher JM, Schachar R (2008) Puppets, robots, critics and actors within a taxonomy of attention for developmental disorders. J Int Neuropsychol Soc 14: 673-690.

12. Willcutt EG, Doyle AE, Nigg JT, Faraone SV, Pennington BF (2005) Validity of the executive function theory of attention-deficit/hyperactivity disorder: a metaanalytic review. Biol Psychiatry 57: 1336-1346.

13. Karalunas SL, Huang-Pollock CL, Nigg JT (2012) Decomposing attentiondeficit/hyperactivity disorder (ADHD)-related effects in response speed and variability. Neuropsychology 26: 684-694.

14. Nigg JT (2005) Neuropsychologic theory and findings in attention-deficit/ hyperactivity disorder: the state of the field and salient challenges for the coming decade. Biol Psychiatry 57: 1424-1435.

15. Abikoff $H$ (1991) Cognitive training in ADHD children: less to it than meets the eye. J Learn Disabil 24: 205-209.

16. Tripp G, Wickens JR (2009) Neurobiology of ADHD. Neuropharmacology 57 579-589.

17. Amodio DM, Master SL, Yee CM, Taylor SE (2008) Neurocognitive components of the behavioral inhibition and activation systems: implications for theories of self-regulation. Psychophysiology 45: 11-19.

18. Chevrier A, Schachar RJ (2010) Error detection in the stop signal task. Neuroimage 53: 664-673

19. Pliszka SR, Glahn DC, Semrud-Clikeman M, Franklin C, Perez R 3rd, et al. (2006) Neuroimaging of inhibitory control areas in children with attention deficit hyperactivity disorder who were treatment naive or in long-term treatment. Am J Psychiatry 163: 1052-1060.

20. Suskauer SJ, Simmonds DJ, Fotedar S, Blankner JG, Pekar JJ, et al. (2008) Functional magnetic resonance imaging evidence for abnormalities in response selection in attention deficit hyperactivity disorder: Differences in activation associated with response inhibition but not habitual motor response. Journal of Cognitive Neuroscience 20: 478-493.

21. Curtis DF (in press) Structured dyadic behavior therapy processes for ADHD intervention. Psychotherapy.

22. Kazdin AE, Whitley MK (2006) Comorbidity, case complexity, and effects of evidence-based treatment for children referred for disruptive behavior. J Consult Clin Psychol 74: 455-467.

23. McDougal JL, Clonan SM, Martens BK (2000) Using organizational change procedures to promote the acceptability of prereferral intervention services: The School-based Intervention Team Project. School Psychology Quarterly 15 149-171.

24. Curtis DF (2010) ADHD symptom severity following participation in a pilot 10-week, manualized, family-based behavioral intervention. Child and Family Behavior Therapy 32: 231-241.

25. Curtis DF, Chapman S, Dempsey J, Mire S (2013) Classroom changes in ADHD symptoms following clinic-based behavior therapy. J Clin Psychol Med Settings 20: 114-122.

26. Barkley RA (1997) Defiant children: A clinicianâ $€^{\mathrm{TM}} \mathbf{S}$ manual for assessment and parent training. (3rdedn), Guilford: New York, USA

27. Cunningham CE (2005) COPE: Large-group, community-based, familycentered parent training. In: Barkley RA (ed.) Attention-deficit hyperactivity disorder: A handbook for diagnosis and treatment. (3rdedn), Guilford Press: New York, USA, 480-498.

28. Cunningham CE (2007) A family-centered approach to planning and measuring the outcome of interventions for children with attention-deficit/hyperactivity disorder. Journal of Pediatric Psychology 32: 676-694. 
29. McMahon RJ, Forehand RL (2005) Helping the non-compliant child: Familybased treatment for oppositional behavior. (2ndedn), Guilford: New York, USA.

30. Sanders MR (1999) The Triple P-Positive parenting program: Towards an empirically validated multilevel parenting and family support strategy for the prevention of behavior and emotional problems in children. Clinical Child and Family Psychology Review 2: 71-90.

31. Barlow DH (2010) Negative effects from psychological treatments: a perspective. Am Psychol 65: 13-20.

32. Gallo KP, Barlow DH (2012) Factors involved in clinician adoption and nonadoption of evidence-based interventions in mental health. Clinical Psychology: Science and Practice 19: 93-106.

33. Chorpita BF (2003) The frontier of evidence-based practice. In: Kazdin AE, Weisz JR (eds.) Evidence-based psychotherapies for children and adolescents. Guilford Press: New York, USA.

34. Eckert TL, Hintze JM (2000) Behavioral conceptualizations and applications of treatment acceptability: Issues related to service delivery and research methodology. School Psychology Quarterly 15: 123-148.
35. Webster-Stratton C, Hammond M (1997) Treating children with early-onset conduct problems: a comparison of child and parent training interventions. J Consult Clin Psychol 65: 93-109.

36. Remschmidt H, Global ADHD Working Group (2005) Global consensus on ADHD/HKD. Eur Child Adolesc Psychiatry 14: 127-137.

37. Pelham WE, Foster EM, Robb JA (2007) The economic impact of attentiondeficit/hyperactivity disorder in children and adolescents. J Pediatr Psychol 32 : 711-727.

38. Wagner SM, McNeil CB (2008) Parent-child interaction therapy for ADHD: A conceptual overview and critical literature review. Child \& Family Behavior Therapy 30: 231-256.

39. [No authors listed] (1999) A 14-month randomized clinical trial of treatment strategies for attention-deficit/hyperactivity disorder. The MTA Cooperative Group. Multimodal Treatment Study of Children with ADHD. Arch Gen Psychiatry 56: 1073-1086.

40. Hinshaw SP (2007) Moderators and mediators of treatment outcome for youth with ADHD: Understanding for whom and how interventions work. Journal of Pediatric Psychology 32: 664-675. 